Article

\title{
Tentative Investigations on Reducing the Edge Effects in Pre-Polishing the Optics
}

\author{
Xiaolong Ke ${ }^{1, *}$, Lei Qiu ${ }^{1}$, Chunjin Wang ${ }^{2, *}$ and Zhenzhong Wang ${ }^{3}$ \\ 1 School of Mechanical and Automotive Engineering, Xiamen University of Technology, \\ Xiamen 361024, China; qiulei@stu.xmut.edu.cn \\ 2 State Key Laboratory of Ultra-precision Machining Technology, Department of Industrial and Systems \\ Engineering, The Hong Kong Polytechnic University, Hung Hom, Kowloon, Hong Kong, China \\ 3 Department of Mechanical and Electrical Engineering, Xiamen University, Xiamen 361005, China; \\ wangzhenzhong@xmu.edu.cn \\ * Correspondence: kexiaolong@xmut.edu.cn (X.K.); chunjin.wang@polyu.edu.hk (C.W.)
}

Received: 24 June 2020; Accepted: 29 July 2020; Published: 30 July 2020

\begin{abstract}
The material removal depth in the pre-polishing stage of the precision optics is usually tens of microns to remove the subsurface damage and grinding marks left by the previous grinding process. This processing of the upstand edge takes a large part of the time at this stage. The purpose of this paper is to develop a method that can reduce the edge effect and largely shorten the processing time of the pre-polishing stage adopting the semirigid (SR) bonnet. The generation of the edge effect is presented based on the finite element analysis of the contact pressure at the edge zone firstly. Then, some experimentations on the edge effect are conducted, and the results proved that the SR bonnet tool can overhang the workpiece edge in the pre-polishing stage to reduce the width and height of the upstand edge to largely shorten the subsequent processing time of it. In addition, there exists a perfect overhang ratio, which generates the upstand edge with the smallest width and height, with no damage to the bonnet tool in the meantime. In addition, one combination of the pre-polishing parameters is concluded according to this method, which can be safely adopted in practical process.
\end{abstract}

Keywords: polishing; edge effect; precision optics; bonnet polishing; semirigid bonnet

\section{Introduction}

Nowadays, computer-controlled optical surfacing (CCOS) technology has been widely used to manufacture the telescope mirrors, optical lens, and molds, etc. [1]. The processed surface form error can usually reach tens of nanometers or even several nanometers. However, there still exists a significant challenge, which is the control of the part edge combined with the speed of processing to enhance the processing accuracy. Unlike ion beam figuring or fluid jet polishing [2], there are edge effects in most CCOS processes such as small tool polishing [3], magnetorheological finishing [4], bonnet polishing [5], and so on. The edge effect would lead to edge-roll, and it needs to be further corrected with smaller tools. It takes a large part of the processing time.

To date, many studies on controlling the edge have been done since the application of CCOS technology. In 1982, Jones [6] firstly adopted the arc raster path to successfully polishing a segmented mirror using the small tool polishing technology, with the initial error $0.0425 \lambda(\lambda=632.8 \mathrm{~nm})$ to the final error $0.012 \lambda$ including the edge. Zhang et al. [7] studied the edge removal profile when the tool overhangs the workpiece edge in small tool polishing, and also presented an edge control algorithm for edge figuring [8]. Cordero-Dávila et al. [9] proposed a skin model to evaluate the polishing pressure at the edge when polishing with a circular tool and correctly predicted that a greater amount of material was removed from the edge of the workpiece. Guo et al. [10] analyzed the 
edge effect of the fluid jet polishing based on the Finite Element Analysis (FEA) method and finally proved that the edge effect could be neglected in this technology. Dae Wook et al. [11] provided a new parametric model to accurately predict the edge Tool Influence Function (TIF) for cases of polishing tools that are either spinning or orbiting over the edge of the workpiece. Liu et al. [12] modeled the edge TIF of computer-controlled active lap polishing based on two methods: one is based on the pressure obtained through FEA, and another is based on a parametric equivalent pressure model. Wan et al. [13] determined the dwell time using a space-variant deconvolution algorithm to control the edge error. Recently, Zhong et al. [14] presented a novel method to reduce the edge effect in the magnetorheological finishing (MRF) through further analyzing the stability of the MRF edge tool influence function (TIF) and correcting the post-edge algorithm of dwell time.

Bonnet polishing is a promising CCOS process that has been successfully used to polish aspheric or freeform surfaces. It also faces the edge effect problem, especially when polishing the large segmented telescope mirrors. There are three main strategies to control the edge of the segmented mirrors presented by Walker et al. [15]: (1) use of sacrificial wasters around the edge; (2) active control of edge-profiles by optimizing polishing process parameters; (3) controlled turning up of the edges during most of the process, followed by a final edge-rectification stage. However, the first strategy was rejected later because of the potential distortion of the surface induced by the adhesive-bonds and the risk with waster attachment and detachment [16]. The combination of strategies (2) and (3) assisting with the modeled edge tool influence function [17] had been used to effectively control the edge in the process-chain for E-ELT segments, which was using the Tool-Lift method generating various sizes of tool influence functions to produce surfaces with gently-sloping peripheral up-stands edges and followed by corrective finishing with smaller tools [18-20]. Beaucamp et al. [21] also did some experimentations on extreme ultraviolet blanks to optimize the polishing parameters over the edge.

The edge specification for the segmented telescope mirrors is very stringent. Different from the telescope mirrors, there are many other optical comments that have a much larger allowable edge for assembly purposes [22]. In other words, the ratio of the allowable edge to the full size is much larger. It is called the LAE optics hereinafter for short. It allows for the downturned edge outside the Effective Aperture (EA). However, the first step of the common procedure of pre-polishing this kind of optics is still polishing the whole surface without any tool overhanging the part edge or over a very tiny distance, leaving a quite wide upstand edge. Then, use a smaller tool to do the corrective polishing of the edge zone until the whole EA can be measured by the interferometer. However, the material removal depth in this stage is usually tens of microns to remove the subsurface damage and grinding marks left by the previous grinding process. The processing of the upstand edge takes a large part of time in the pre-polishing stage. With this in view, investigations on reducing the edge effects were made to find a method using a semirigid (SR) bonnet $[23,24]$, which can largely shorten the pre-polishing time. Section 2 analyzed the edge effect theoretically, including explaining the generation of the edge effect, finite element analysis of the contact pressure, and one hypothesis that can implement the purpose of this paper. Experiments were conducted in Section 3 to test this hypothesis. In addition, validation experiments were conducted in Section 4 together with the discussion.

\section{Theoretical Analysis of the Edge Effect}

\subsection{Generation of the Edge Effect}

The material removal in bonnet polishing could be controlled to be deterministic and its amount $H(x, y)$, which is equal to the two-dimensional convolution between the material removal function per unit time $R(x, y)$ and the dwell time function $D(x, y)$, along with the tool path, can be expressed as [25]:

$$
H(x, y)=R(x, y) * * D(x, y) .
$$


Therefore, the surface residual error $E(x, y)$ after the polishing process can be expressed as:

$$
E(x, y)=H_{0}(x, y)-H(x, y) .
$$

where $H_{0}(x, y)$ is the desired material removal.

Uniform material removal simulations regarding the edge have been conducted based on Equations (1) and (2). Figure 1 shows the geometrical model when the bonnet tool overhangs the edge. The overhang ratio $\tau$ is the ratio of overhang distance $\omega$ to the diameter of the contact spot $\mathrm{D}$. The assumption that the TIF over the whole surface is all the same including the edge zone is made in this simulation. The initial error surface is an $80 \mathrm{~mm} \times 80 \mathrm{~mm}$ standard flat surface. Gaussian-like tool influence function with a diameter of $10 \mathrm{~mm}$ was adopted, as shown in Figure 2. Its peak removal rate is $3.0 \mu \mathrm{m} / \mathrm{min}$. The overhang ratio varies from 0 to 1 , with an increment of 0.1 . The distance in $x$ - and $y$-directions between each dwell point is $1 \mathrm{~mm}$. In addition, the dwell time at each dwell point is $1 \mathrm{~s}$.

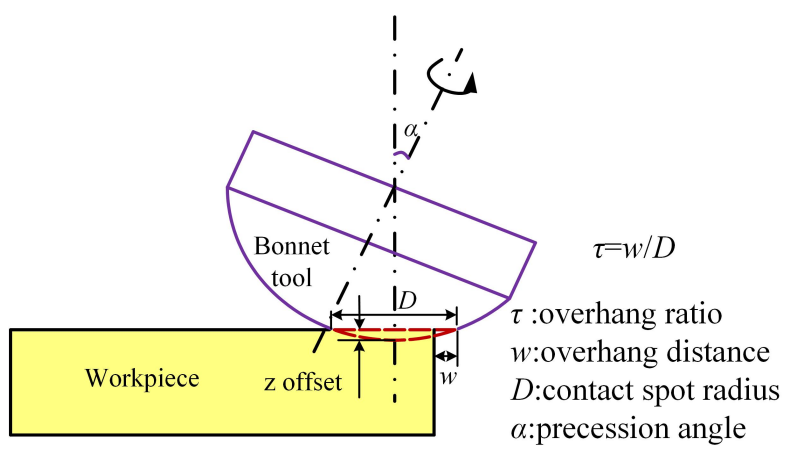

Figure 1. Modeling of the bonnet tool polishing overhanging the edge.

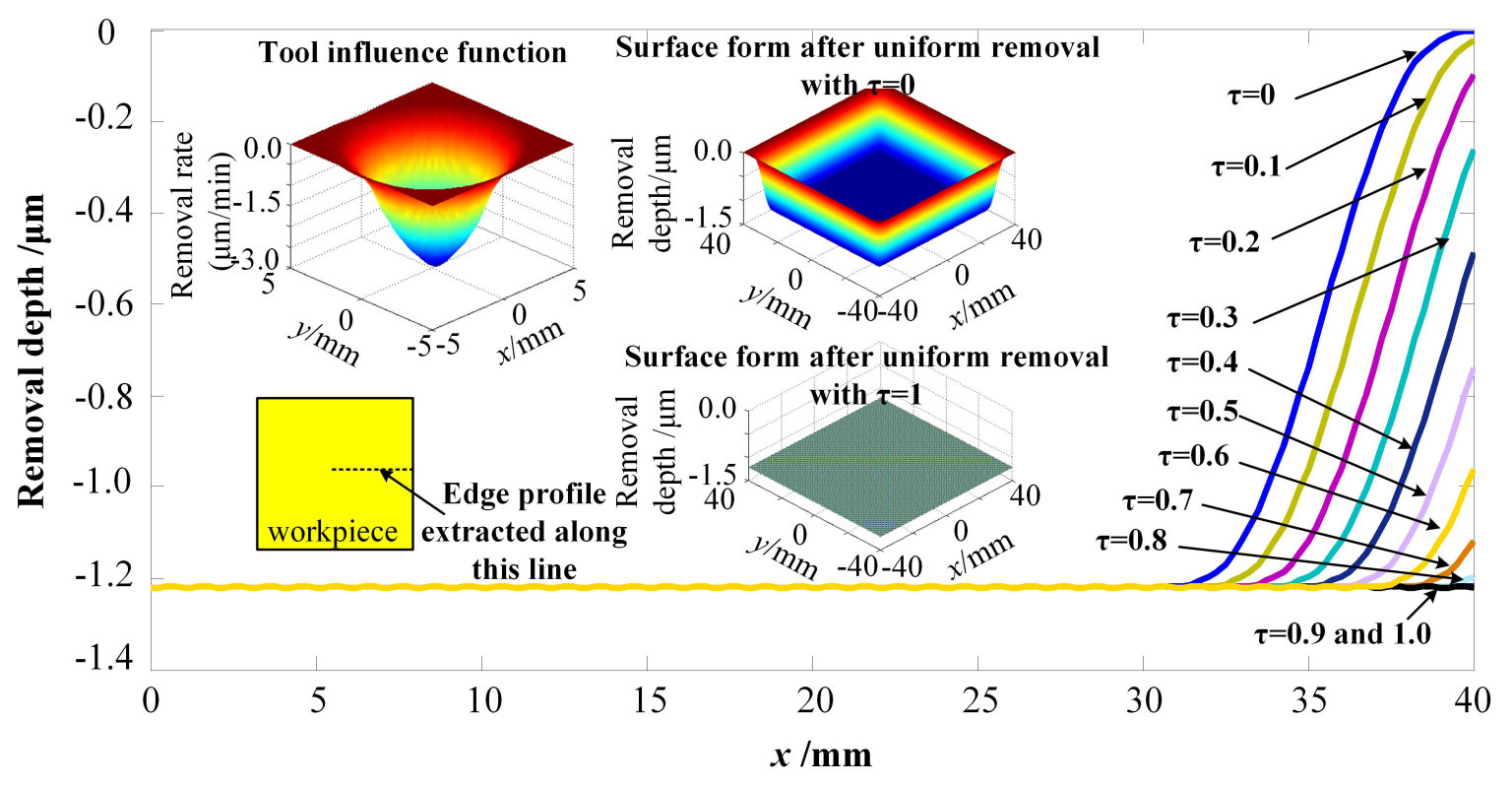

Figure 2. Edge profile after uniform polishing with different overhang ratios.

Figure 2 shows the simulation results of this group of uniform removal based on Equations (1) and (2). When $\tau=0$, the height and the width of the upstand edge are the largest, as shown in the sectional profile and 3D profile in Figure 2. The edge width is the diameter of TIF. When $\tau=1$, which means that the bonnet tool is totally out of the workpiece, there is no edge upstand in the simulation result. This simulation demonstrates the removal results when there is no edge effect. However, in the practical manufacturing process, the shape of the tool influence function at the 
edge zone is irregular due to the change of the contact pressure when the bonnet tool compresses the edge [17]. In addition, the bonnet tool is impossible to totally overhang the edge thoroughly $(\tau=1)$, which will damage the tool. This is the main reason for the generation of the edge effect.

\subsection{Finite Element Analysis of the Contact Pressure}

To further understand the variation of the contact pressure, finite element analysis of the contact pressure under different overhang ratios has been done. Figure 3a shows the simulated displacement result when $\tau=0.5$. Half of the model was built to simplify the simulation model. The bonnet tool was simplified, just leaving the spherical part. The bonnet radius is $80 \mathrm{~mm}$, and the workpiece material is BK7. In the simulation process, the bonnet tool was fixed by constraining all DOFs of the fringe area, and the workpiece was defined to move upward with $1.25 \mathrm{~mm}$ to deliver a $28 \mathrm{~mm}$ diameter contact spot (The size is calculated when the spot is in the central zone.) In addition, the inflated pressure loaded on the inner surface of the bonnet tool is $0.25 \mathrm{MPa}$. The overhang ratio $\tau$ varies from 0 to 0.5 in the simulation, with an increment of 0.1. Other detailed simulation parameters had been reported in our previous publications [23,26].

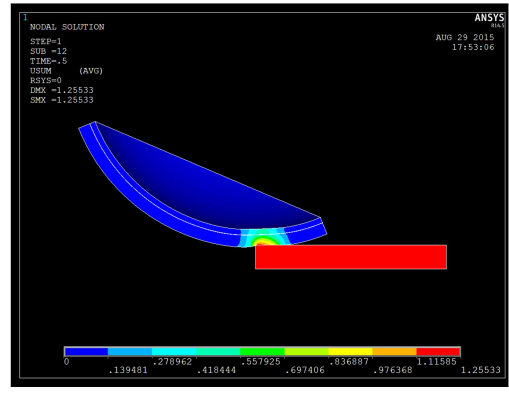

(a)

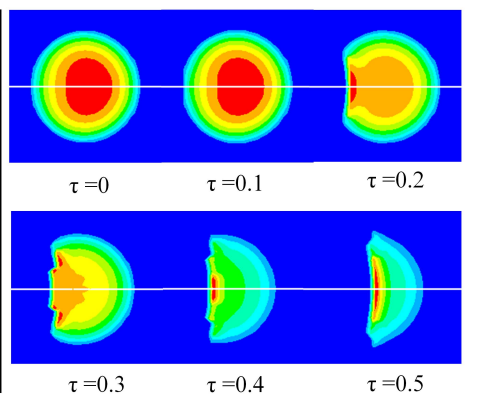

(b)

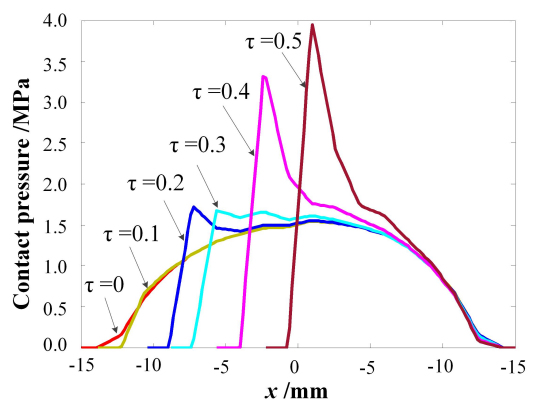

(c)

Figure 3. Finite element modeling of the contact pressure in the edge zone with different overhang ratio. (a) finite element model and corresponding displacement deformation result when $\tau=0.5$; (b) simulation results of the contact pressure distribution shape with different $\tau$; (c) sectional profile of the contact pressure along the white line plotted in (b).

When $\tau=0.5$, the finite element model and corresponding displacement deformation result are presented in Figure 3a. Figure 3b shows the contact pressure distribution shapes under this different $\tau$, and their corresponding sectional profiles have also been extracted, as shown in Figure 3c. The sharp increase of the contact pressure turns out at the edge zone, and its peak value becomes more significant with the rise of $\tau$.

\subsection{Discussion on the Edge Error Profile}

The sharp increase of the contact pressure at the edge area would lead to the dramatic increase of material removal there. It makes the material removal become unpredictable at the edge zone. As shown in Figure 4, there are three cases of the error profile at the edge zone after polishing using the bonnet tool according to the reported results by Walker et al. [16] and Li et al. [19], which are the profile with only the upturned (case 1), the profile with upturned and downturned (case 2), and the profile with only the downturned (case 3), respectively. When polishing the segmented mirrors, the downturned edge profile is unacceptable. However, in the case of polishing LAE optics, a downturned edge is acceptable if the downturned zone is outside EA. Hence, a hypothesis can be made that there exists a perfect $\tau$ (case 4 , as shown in Figure 4 ), which makes the edge profile have the smallest PV value and edge width, without damaging the bonnet tool in the meantime. It would be perfect for the pre-polishing of LAE optics. In case 4, no complicated control of the tool-lift will be needed anymore. Generally, the gradient of the upstand edge is too high to be measured using the interferometer after a few pre-polishing steps. Many corrective polishing iterations on the edge zone are needed to reduce its 
gradient. However, this hypothesis gives the hope for pre-polishing LAE optics to the accuracy level, which can be measured by the interferometer within just in a few iterations. If large size TIF is used to enhance the pre-polishing efficiency firstly, a part of the upstand edge zone may be in EA as shown in Figure 4. Nevertheless, its height and width are much smaller compared to cases 1 and 2, which dramatically reduces the processing time in the following step. If relatively small size TIF is used, the upstand edge zone may be outside EA. Then, there will be no need for corrective polishing the edge in this case. Hence, if this hypothesis can be implemented, it will dramatically reduce the edge processing time in the pre-polishing stage, and largely shorten the manufacturing cycle of LAE optics.

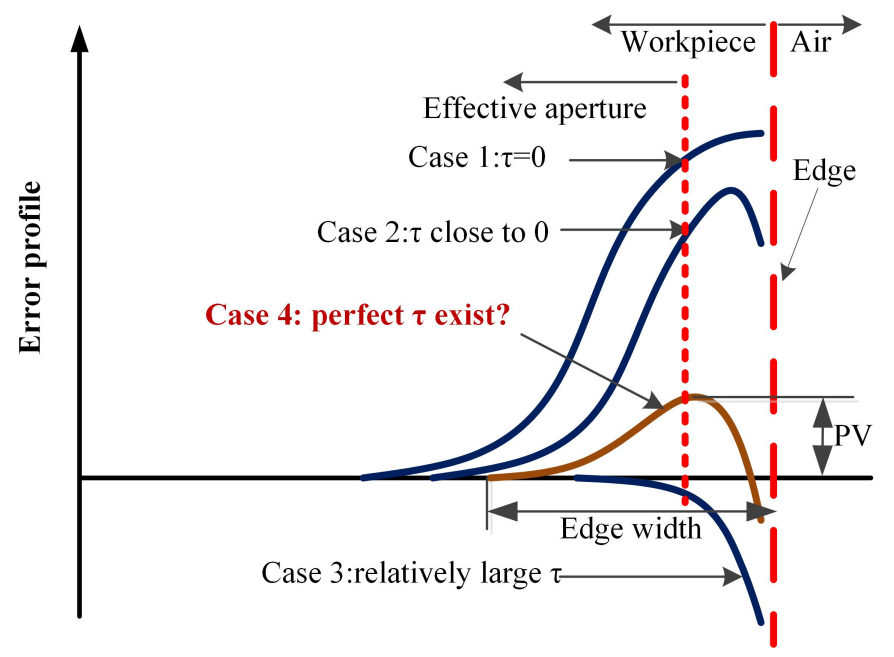

Figure 4. Demonstration of several practical edge effect cases about the error profile of the edge.

\section{Experiments}

Experiments have been conducted to test this hypothesis in this paper. The strategy of the experiment is to polish several lines with different overhang ratio $\tau$ to compare their edge profiles. Figure 5 shows the experimental prototype. R80 SR bonnet tool was used in this experiment. Considering that the TIF with a diameter of $28 \mathrm{~mm}$ of R80 SR bonnet has high material removal efficiency and stability, and Gaussian-like shape, it is mostly adopted in the practical pre-polishing process. Hence, TIF with the diameter of $28 \mathrm{~mm}$ was also adopted in this experiment. The polishing experiment was done on a flat surface made of fused silica, with the size of $200 \mathrm{~mm} \times 200 \mathrm{~mm}$. The flat surface had been finely polished before this experiment. Considering that the polishing pad would be rapidly damaged when the overhang ratio is larger than 0.5 , the overhang ratio adopted in these experiments varied from 0 to 0.5 . Other experimental conditions have been shown in Table 1 . In total, 11 lines were polished in this experiment, and the length of each line was about $85 \mathrm{~mm}$.

Table 1. Experimental conditions.

\begin{tabular}{cc}
\hline Conditions & Value \\
\hline Precession angle(deg) & 23 \\
Inner pressure(MPa) & 0.25 \\
H-axis speed $(\mathrm{rpm})$ & 1500 \\
Feeding speed $(\mathrm{mm} / \mathrm{min})$ & 100 \\
Radius of bonnet $(\mathrm{mm})$ & 80 \\
Polishing slurry & $\sim 2 \mathrm{wt} \% \mathrm{CeO}_{2}$ \\
Overhang ratio $\tau$ & $0-0.5$, with the increment of 0.05 \\
\hline
\end{tabular}




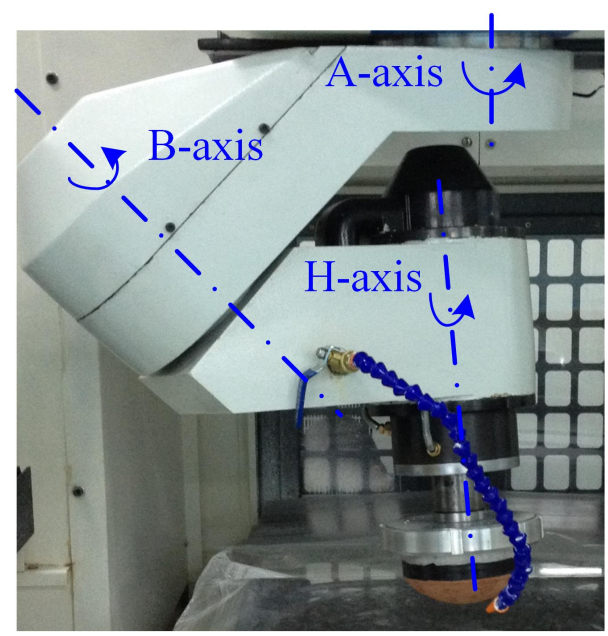

Figure 5. Experimental prototype.

The measured results using the interferometer have been shown in Figure 6. Figure 6a shows the removed form shape of each polishing line (Around the half line close to the edge has been shown, and some data were missing due to the large gradient of the form error). It indicates that the width of the upstand edge becomes smaller with the increasing $\tau$. To compare these profiles quantitatively, six sectional profiles among them were extracted as shown in Figure 6b. It hints that both the width and height of the upstand edge become smaller with the increasing $\tau$. When $\tau=0$, the width of the upstand edge was $\sim 23 \mathrm{~mm}$ and the height is $\sim 4.2 \lambda$. When $\tau=0.5$, its width and height dramatically reduced to $\sim 8 \mathrm{~mm}$ and $\sim 1.25 \lambda$, respectively. Hence, the hypothesis made in part 2 is proved to be correct. With this in view, in the pre-polishing process of optical surfaces using the SR bonnet, the tool can overhang the workpiece and the adopted overhang ratio can be increased to a threshold without damaging the polishing pad.

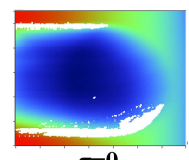

$\boldsymbol{\tau}=\mathbf{0}$

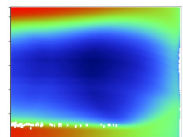

$\tau=\mathbf{0 . 2 0}$

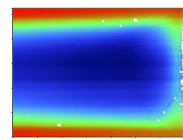

$\tau=\mathbf{0 . 4 0}$
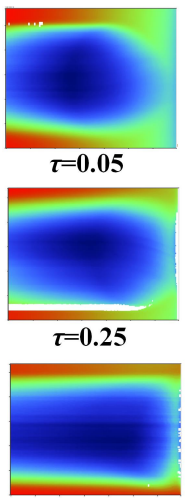

$\tau=\mathbf{0 . 4 5}$

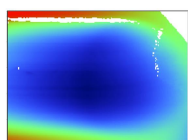

$\tau=\mathbf{0 . 1 0}$

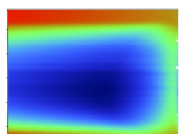

$$
\tau=\mathbf{0 . 3 0}
$$

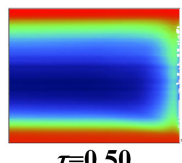

(a)

$\tau=\mathbf{0 . 5 0}$

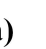

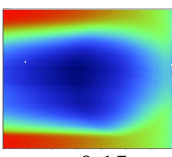
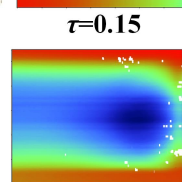

$\tau=\mathbf{0 . 3 5}$

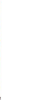

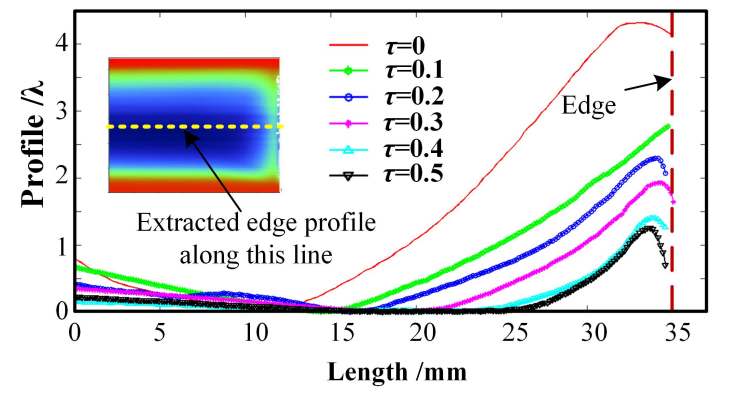

(b)

Figure 6. Experiment results of each polishing line. (a) removed form shape of each polishing line and (b) extracted sectional profiles of them. $(\lambda=632.8 \mathrm{~nm})$.

\section{Verification and Discussion}

Considering that only one line was polished under each condition in the experiments conducted in Section 3, and the bonnet tool deals with the edge only at the end of each line within an extremely short time, it cannot reflect that the bonnet tool can not be damaged after polishing the whole surface under these corresponding conditions. Hence, several verification experiments were also conducted to test the conclusion summarized above. Experiments have been done on two ground flat fused silica glasses with the size of $100 \mathrm{~mm} \times 100 \mathrm{~mm}$. 
Firstly, the pre-polishing process was executed using the conditions as shown in Table 1, except for the feeding speed being $800 \mathrm{~mm} / \mathrm{min}$. The raster path with the pitch of $2 \mathrm{~mm}$ was adopted. We had optimized the structure of the SR bonnet as reported in Ref. [24]. There are two kinds of SR bonnets with two different embedded materials, which are steel sheet and steel mesh, respectively. The embedded material of the one used in the experiment above was a steel sheet, which has higher rigidity than the one embedded with steel mesh, leading to larger contact pressure. In this experiment, we also used the R80 SR bonnet embedded with the steel sheet. After 24 min polishing of the whole surface, the polyurethane pad was heavily worn as shown in Figure 7a. In addition, the R80 SR bonnet embedded with the steel mesh was also tested. Considering that it has less rigidity, the polishing spot with $25 \mathrm{~mm}$ size and with the $\mathrm{H}$-axis speed $1000 \mathrm{rpm}$ were adopted. When the bonnet tool contacted the edge of the glass, the pad material was directly removed, just like the turning process as shown in Figure $7 \mathrm{~b}$. Hence, the conclusion made above is not suitable for the SR bonnet embedded with the steel mesh. The R80 SR bonnet tool embedded with steel sheet was adopted in the subsequent experiments.

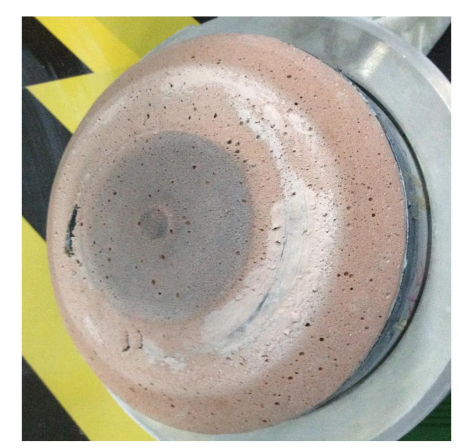

(a)

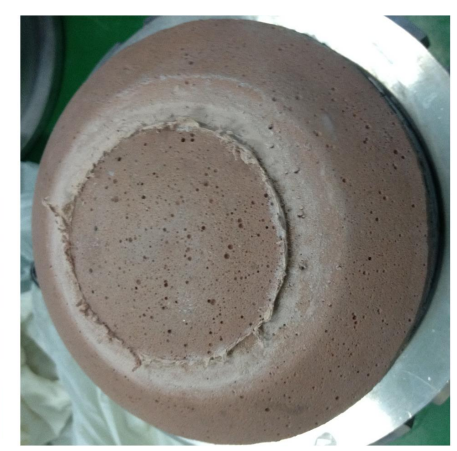

(b)

Figure 7. Two different SR bonnet tools with the damaged polyurethane pad. (a) damaged SR bonnet tool with the embedded material of stainless steel sheet and (b) damaged SR bonnet tool with the embedded material of steel mesh.

Because the polishing pad was damaged under the conditions as shown in Table 1, the size of the polishing spot was reduced to $25 \mathrm{~mm}$ in the following experiments. In addition, three cycles of pre-polishing on one $100 \mathrm{~mm} \times 100 \mathrm{~mm}$ surface were executed with three different $\mathrm{H}$-axis speeds of $1000 \mathrm{rpm}, 1250 \mathrm{rpm}$, and $1500 \mathrm{rpm}$, respectively. The polishing time of each cycle was still 24 min. The H-axis speed started with $1000 \mathrm{rpm}$. After the first cycle, no obvious wear was found on the polishing pad. After polishing with $1250 \mathrm{rpm} \mathrm{H}$-axis speed, it was still hard to find the obvious tool wear. However, after polishing with $1500 \mathrm{rpm} \mathrm{H}$-axis speed, the polishing pad became worn as shown in Figure 8. It indicates that the threshold value for the $\mathrm{H}$-axis speed lies between $1250 \mathrm{rpm}$ and $1500 \mathrm{rpm}$ under those conditions. After these three pre-polishing times, the grey of the ground surface has been cleared and it also reached an accuracy level that could be measured through the interferometer. Figure 9 shows the measured result of the polished surface. The size of the area with the effective measured data reaches $\sim 84.5 \mathrm{~mm} \times 83 \mathrm{~mm}$. In addition, the average upstand width of the edge is only $\sim 8 \mathrm{~mm}$. Through this experiment, the $\mathrm{H}$-axis speed of $1250 \mathrm{rpm}$ when the polishing spot size is $25 \mathrm{~mm}$ (other conditions are as shown in Table 1) can be adopted safely in the practical pre-polishing process when using the R80 SR bonnet. 


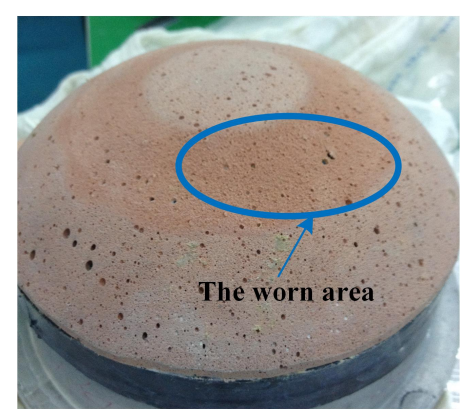

Figure 8. The polishing pad becomes worn when the $\mathrm{H}$-axis speed reaches $1500 \mathrm{rpm}$ with the polishing spot size of $25 \mathrm{~mm}$.

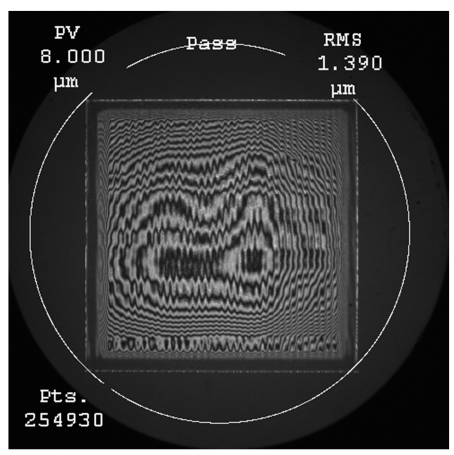

(a)

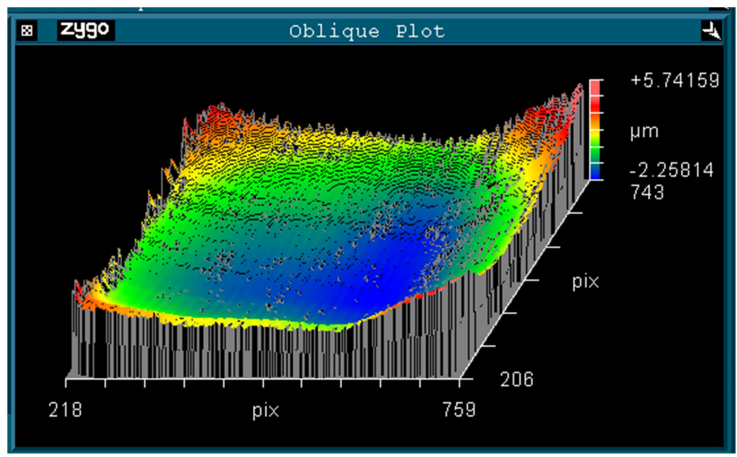

(b)

Figure 9. Measured results of the surface after three times of pre-polishing. (a) interference fringe image and (b) surface form error result.

If the above surface was pre-polished without the overhang of the tool, the upstand edge width would be $25 \mathrm{~mm}$ (equal to the diameter of the polishing spot as demonstrated in Figure 2). Though the edge width with $8 \mathrm{~mm}$ is larger than the allowable edge used in LAE optics when the part size is $100 \mathrm{~mm} \times 100 \mathrm{~mm}$, it can still largely reduce the subsequent corrective polishing time of the edge in the pre-polishing stage. When the workpiece is enlarged to $400 \mathrm{~mm} \times 400 \mathrm{~mm}$, this edge width will be allowable. In addition, the corrective polishing of the edge zone will not be needed in this situation. It will dramatically shorten the polishing cycle of the LAE optics, especially when encountering the large cases.

\section{Conclusions}

Tentative investigations on reducing the edge effect of adopting the SR bonnet have been done in this paper. The experimental results proved that the SR bonnet tool can overhang the workpiece edge in the pre-polishing stage to reduce the width and height of the upstand edge aiming to largely shorten the subsequent processing time of it. In addition, there exists a perfect overhang ratio (with the same polishing spot size used in the central zone) which generates the upstand edge with the smallest width and height, with no damage to the bonnet tool in the meantime. No more complicated Tool-Lift control will be needed in this method. The pre-polishing process of LAE optics adopting this method would largely shorten its manufacturing cycle, especially at the polishing stage. In addition, one combination of the polishing parameters for R80 SR bonnet according to this method has been concluded, which is: overhang ratio $=0.5$, TIF diameter $=25 \mathrm{~mm}, \mathrm{H}$-axis speed $=1250 \mathrm{rpm}$, precession angle $=23 \mathrm{deg}$, and Inner pressure $=0.25 \mathrm{MPa}$. 
Author Contributions: X.K. and C.W. contributed to the main idea of the paper and wrote the manuscript. X.K. and L.Q. analyzed the data. Z.W. provided valuable suggestions for the manuscript. C.W. performed the experiments and fully polished the language. All authors discussed the results and commented on the manuscript. All authors have read and agreed to the published version of the manuscript.

Funding: This work is supported by the National Science Foundation of China (NSFC) under Grant No. 51275433 and the Natural Science Foundation of Fujian Province, China under Grant No. 2018J01528.

Conflicts of Interest: The authors declare no conflict of interest.

\section{References}

1. Zhang, D.; Guo, H. A trajectory planning method for polishing optical elements based on a non-uniform rational B-spline curve. Appl. Sci. 2018, 8, 1355. [CrossRef]

2. Han, Y.; Zhang, L.; Fan, C.; Zhu, W.; Beaucamp, A. Theoretical study of path adaptability based on surface form error distribution in fluid jet polishing. Appl. Sci. 2018, 16, 1814. [CrossRef]

3. Jones, R.A. Optimization of computer controlled polishing. Appl. Opt. 1977, 16, 218-224. [CrossRef] [PubMed]

4. Kordonski, W.; Gorodkin, S. Material removal in magnetorheological finishing of optics. Appl. Opt. 2011, 50, 1984-1994. [CrossRef] [PubMed]

5. Walker, D.D.; Brooks, D.; King, A.; Freeman, R.; Morton, R.; McCavana, G.; Kim, S.-W. The 'Precessions' tooling for polishing and figuring flat, spherical and aspheric surfaces. Opt. Express 2003, 11, 958-964. [CrossRef]

6. Jones, R.A. Segmented mirror polishing experiment. Appl. Opt. 1982, 21, 561-564. [CrossRef]

7. Zhang, X.; Yu, J.; Sun, X. Theoretical method for edge figuring in computer-controlled polishing of optical surface. Proc. SPIE 1994, IV, 239-246. [CrossRef]

8. Xuejun, Z.; Jingchi, Y.; Xiafei, S. Edge control in computer controlled optical polishing. Proc. SPIE 1995, 12536, 239-242. [CrossRef]

9. Cordero-Davila, A.; Gonzalez-Garcia, J.; Pedrayes-Lopez, M.; Aguilar-Chiu, L.A.; Cuautle-Cortes, J.; Robledo-Sanchez, C. Edge effects with the Preston equation for a circular tool and workpiece. Appl. Opt. 2004, 43, 1250-1254. [CrossRef]

10. Guo, P.; Fang, H.; Yu, J. Edge effect in fluid jet polishing. Appl. Opt. 2006, 45, 6729-6735. [CrossRef]

11. Kim, D.W.; Park, W.H.; Kim, S.-W.; Burge, J.H. Parametric modeling of edge effects for polishing tool influence functions. Opt. Express 2009, 17, 5656-5665. [CrossRef] [PubMed]

12. Liu, H.; Wu, F.; Zeng, Z.; Fan, B.; Wan, Y. Edge effect modeling and experiments on active lap processing. Opt. Express 2014, 22, 10761-10774. [CrossRef]

13. Wan, S.; Zhang, X.; Wang, W.; Xu, M.; Jiang, X. Edge control in precision robotic polishing based on space-variant deconvolution. Precis. Eng. 2019, 55, 110-118. [CrossRef]

14. Zhong, X.; Fan, B.; Wu, F. Reducing edge error based on further analyzing the stability of edge TIF and correcting the post-edge algorithm in MRF process. Opt. Rev. 2020, 27, 14-22. [CrossRef]

15. Walker, D.; Beaucamp, A.; Dunn, C.; Evans, R.; Freeman, R.; Morton, R.; Wei, S.; Yu, G. Active control of edges and global microstructure on segmented mirrors. Proc. SPIE 2008, 7018, 701812. [CrossRef]

16. Walker, D.; Yu, G.; Li, H.; Messelink, W.; Evans, R.; Beaucamp, A. Edges in CNC polishing: from mirror-segments towards semiconductors, paper 1: Edges on processing the global surface. Opt. Express 2012, 20, 19787-19798. [CrossRef] [PubMed]

17. Li, H.; Yu, G.; Walker, D.; Evans, R. Modelling and measurement of polishing tool influence functions for edge control. J. Eur. Opt. Soc. Rap. Pub 2011, 6, 1104801-1104806. [CrossRef]

18. Walker, D.; Beaucamp, A.; Evans, R.; Fox-Leonard, T.; Fairhurst, N.; Gray, C.; Hamidi, S.; Li, H.; Messelink, W.; Mitchell, J. Edge-control and surface-smoothness in sub-aperture polishing of mirror segments. Proc. SPIE 2012, 8450, 84502A. [CrossRef]

19. Li, H.; Walker, D.; Yu, G.; Sayle, A.; Messelink, W.; Evans, R.; Beaucamp, A. Edge control in CNC polishing, paper 2: simulation and validation of tool influence functions on edges. Opt. Express 2013, 21, 370-381. [CrossRef]

20. Yu, G.; Walker, D.; Li, H.; Zheng, X.; Beaucamp, A. Research on edge-control methods in CNC polishing. J. Eur. Opt. Soc. Rap. Pub. 2017, 13, 1-13. [CrossRef] 
21. Beaucamp, A.; Namba, Y.; Charlton, P. Corrective finishing of extreme ultraviolet photomask blanks by precessed bonnet polisher. Appl. Opt. 2014, 53, 3075-3080. [CrossRef] [PubMed]

22. Paul, R.; Yoder, J. Mounting Optics in Optical Instruments; SPIE Press: Bellingham, WA, USA, 2008.

23. Wang, C.; Yang, W.; Wang, Z.; Yang, X.; Sun, Z.; Zhong, B.; Pan, R.; Yang, P.; Guo, Y.; Xu, Q. Highly efficient deterministic polishing using a semirigid bonnet. Opt. Eng. 2014, 53, 095102. [CrossRef]

24. Wang, C.; Wang, Z.; Wang, Q.; Ke, X.; Zhong, B.; Guo, Y.; Xu, Q. Improved semirigid bonnet tool for high-efficiency polishing on large aspheric optics. Int. J. Adv. Manuf. Technol. 2017, 88, 1607-1617. [CrossRef]

25. Wang, C.; Yang, W.; Wang, Z.; Yang, X.; Hu, C.; Zhong, B.; Guo, Y.; Xu, Q. Dwell-time algorithm for polishing large optics. Appl. Opt. 2014, 53, 4752-4760. [CrossRef]

26. Ke, X.; Wang, C.; Guo, Y.; Xu, Q. Modeling of tool influence function for high-efficiency polishing. Int. J. Adv. Manuf. Technol. 2016, 84, 2479-2489. [CrossRef]

(C) 2020 by the authors. Licensee MDPI, Basel, Switzerland. This article is an open access article distributed under the terms and conditions of the Creative Commons Attribution (CC BY) license (http://creativecommons.org/licenses/by/4.0/). 